\title{
MODELING REMOVAL OF ACCUMULATED POTASSIUM FROM T-TUBULES BY INWARD RECTIFIER POTASSIUM CHANNELS
}

\author{
Willemien Wallinga**, Maarten Vliek*, Eduard D. Wienk*, Martin J. Alberink*, Dirk L. Ypey ${ }^{\square}$ \\ * Institute for BioMedical Technology, Department of Electrical Engineering, University of Twente, \\ P.O. Box 217, 7500 AE Enschede, The Netherlands. \\ \# e-mail address: wlg@el.utwente.nl \\ ${ }^{\square}$ Department of Physiology, Leiden University, Leiden, The Netherlands.
}

\begin{abstract}
The membrane models of Cannon et al. [1] and Alberink et al. [2] for mammalian skeletal muscle fibers are based upon Hodgkin-Huxley descriptions of sodium, potassium delayed rectifier and leak conductance's and the capacitive current taking into account fast inactivation of sodium channels. Now inward rectifier and chloride ion currents, sodiumpotassium pump and slow inactivation of sodium channels have been inserted. The behavior of the model with respect to resting membrane potential and action potential firing does not differ remarkably from [2]. The model has been used to study the removal of potassium from the T-tubular space. The inward rectifier current significantly contributes to the restoration of the normal potassium concentration after prolonged action potential firing. This may be an important mechanism to avoid muscle fatigue.
\end{abstract}

\section{INTRODUCTION}

The phenomena in muscular diseases often result from genetic defects in ion channels. This makes it of interest to study the effects of ion channel changes in models of muscular diseases, although the limits of modeling have to be recognized. The number of ion channel types incorporated in membrane models of skeletal muscle fibres is still limited. Therefore, it is useful to use experimental data to insert new components in HodgkinHuxley models of the skeletal muscle fiber membrane [1].

In a muscle fiber a series of action potentials will result in elevation of the T-tubular concentration of potassium. After extension of our previous model [2] with important components for the resting membrane potential we tested the transport of potassium back into the sarcoplasm.

\section{METHODS}

A model of the electrical behavior was taken from [2]. The model contained for both sarcolemma and T-tubule membrane the capacitive current and conductance's for delayed rectifier, regenerative sodium and leak. The intracellular and the $\mathrm{T}$-tubular volumes were space clamped. The coupling between the T-tubular system and the extracellular medium was modeled by an access resistance. With this model current-voltage (I-V)-curves in the quasi-steady state and action potentials were investigated. In the extended model potassium conductance resulted from both delayed and inward rectifier potassium channels [3]. The conductance of the inward rectifier was equal for both membranes [4], while all other conductance's were lower for the T-tubular membranc. Slow inactivation of the regenerative sodium conductance was inserted according to [5]. Instead of a global leak current a chloride conductance was modeled [6], together with a voltage dependent Na-K pump [7]. The diffusion time constant for potassium from the Ttubule to the extracellular medium was $350 \mathrm{~ms}$ [1].

We formulated four criteria to be fulfilled to accept the simulated data. The chloride conductance should account for about $80 \%$ of the total membrane conductance at rest. There should be only one resting membrane potential. The action potential should have a normal duration and amplitude. The potassium accumulation in the T-tubules caused by one action potential should be $0.4 \mathrm{mM}$, e.g. [8].

\section{RESULTS}

The resting membrane potential of the extended model was normal for a set of parameters within the ranges given in literature.

When varying the surface ratio of T-tubular and sarcolemmal membranes (range 1.5 to 4.5 ) the best action potentials were found for 1.5 . This value was used further. The resting membrane potential was $-80 \mathrm{mV}$. The action potential had a rising phase of $1.6 \mathrm{~ms}$, an amplitude of $110 \mathrm{mV}$ and a total duration of $5 \mathrm{~ms}$.

An exarnple of potassium accumulation and removal at a single action potential is plotted in fig. la. Delayed rectifier channels opened gradually after depolarization and gave a singly peaked transient. Inward rectifier current was doubly peaked, because this current increased in the potential range from -80 to $-60 \mathrm{mV}$ and decreased at less negative membrane potential (fig. 1b). The small persistent Na-K pump current and diffusion removed slowly potassium to the original concentration. 

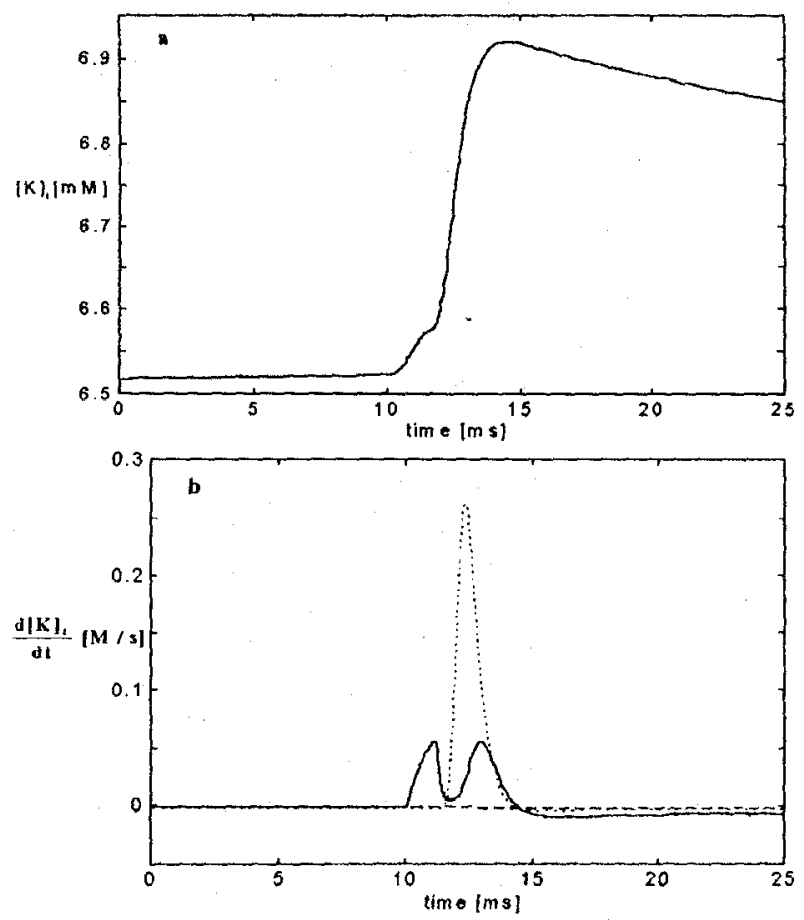

Fig. 1 Transients for a single action potential starting at $10 \mathrm{~ms}$. a. T-tubular potassium concentration, $b$. T-tubular potassium concentration changes caused by the inward rectifier (bold line), delayed rectifier (dotted line) and $\mathrm{Na}-\mathrm{K}$ pump (dashed line)

The removal of potassium from the T-tubules is much quicker in the presence of inward rectifier channels (fig. 2a) than with diffusion only. The ion currents of importance during the removal process were the inward rectifier and chloride currents (fig. $2 b$ ). The membrane potentials of sarcolemmal and T-tubular membranes (not shown) started at $-46 \mathrm{mV}$, a value belonging to $30 \mathrm{mM}$ potassium. The potential of the sarcolemma shifted immediately because of the extracellular potassium concentration of $6.4 \mathrm{mM}$. A smaller change in the Ttubular potential followed. The steps in potentials give the early quick changes in fig. $2 \mathrm{~b}$. Thereafter the potentials gradually shifted to the normal value within $300 \mathrm{~ms}$.

\section{DISCUSSION AND CONCLUSIONS}

We tested the sensitivity of the model behavior for the surface ratio of T-tubular and sarcolemmal membranes, because its value is uncertain. Only the lowest value in the range resulted in acceptable action potentials, while various parameter combinations have been examined.

Elevation of potassium concentration in the $T$-tubules was mainly caused by the delayed rectifier, but in the removal process the inward rectifier was important. The extended model with physiologically acceptable conductance's for inward rectifier and chloride allows simulations of normal resting membrane potentials and action potentials. This model describes a mechanism for enhanced removal of potassium from the T-tubules.
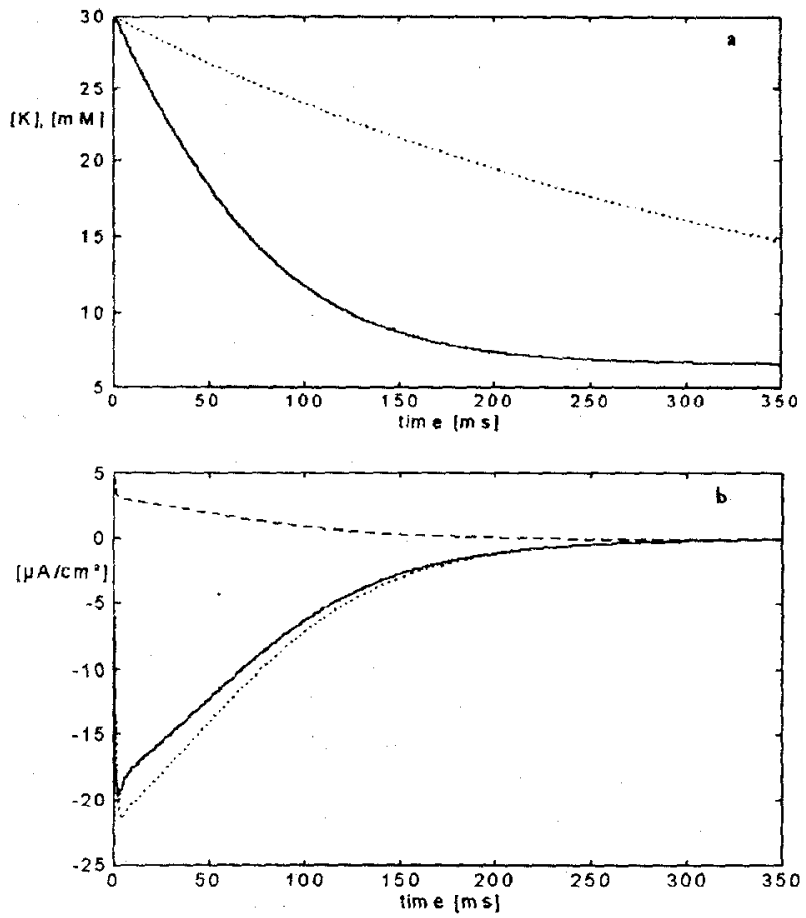

Fig.2. Transients after prolonged firing of action potentials. a. potassium concentration with inward rectificr and chloride currents (bold line) and with diffusion flow only (dashed line), b. inward rectifier (dotted line), chloride (dashed line) and total (solid line) currents per $\mathrm{cm}^{2}$ T-tubular membrane.

\section{REFERENCES}

[1] Cannon, S.C., Brown, R.H., Corey, D.P., "Theoretical reconstruction of myotonia and paralysis caused by incomplete inactivation of sodium channels", Biophys. J., voi. 65, pp. 270-288, 1993.

(2) Alberink, M.J., Wallinga, W., Wolters, H., Ypey, D.L., Links, T.P., Boom, H.B.K., "Theoretical analysis of human muscle membrane behavior in hypokalemic periodic paralysis", IEEE-EMBS Proceedings, Montreal, 2pp, 1995.

[3] Standen, N.B., Stanfield, P.R., "Inward rectification in skeletal muscle: a blocking particle model", Pfligers Arch., vol. 378, pp. 173-176, 1978.

[4] Ashcroft, F.M., Heiny, J.A., Vergara, J., "Inward rectification in the transversetubular system of frog skeletal muscle studied with potentiometric dyes", J. Physiol., vol. 359, pp. 269-291, 1985.

[5] Ruff, R.L., Simoncini, L., Stühmer, W., "Slow sodium channels inactivation in mammalian muscle: a possible role in regulating excitability", Muscle \& Nerve, vol. 11, pp. 502-510, 1988.

[6] Pusch, M., Steinmeyer, K., Jentsch, T.J., "Low single channel conductance of the major skeletal muscle chloride channel, ClC-1", Biophys. J., vol. 66, pp. 149-152, 1994.

[7] Siegenbeek van Heukelom, J., "The role of the potassium inward rectifier in defining cell nembrane potentials in low potassium media, analyzed by computer simulation", Biophys. Chem., vol. 50, pp. 345-360, 1994.

[8] Kirsch, G.E., Nichols, R.A., Nakajima, S., "Delayed rectification in the transverse tubules", J. Gen. Physiol, vol. 70, pp. 1-21, 1977. 